\title{
Le Règlement d'exécution de la FMH est définitivement adopté
}

En décembre 2006, le temps avait manqué pour débattre du Règlement d'exécution (RE) de la FMH, qui contient les principales dispositions d'exécution relatives aux nouveaux statuts, et la Chambre médicale avait donc mis le RE en vigueur à titre provisoire sans l'examiner en détail. Le 3 mai de cette année, elle a pu se consacrer à cet objet. L'orientation générale du document était incontestée et seules quelques propositions de modification ou de complément figuraient à l'ordre du jour. Par 144 voix et 2 abstentions, la Chambre médicale a adopté à l'unanimitéle RE révisé. Le même jour, elle a également accepté à l'unanimité, par 108 voix et 4 abstentions, le Règlement de la Commission de gestion proposé par cette dernière.

Une nouvelle étape de la réforme des structures de la FMH est ainsi terminée:

- L'Assemblée des délégués $(A D)$, qui a déjà siégé trois fois en 2007, est entrée en scène entre la Chambre médicale et le Comité central. L'AD devrait rendre la FMH plus apte à agir et donner une plus large assise au travail du Comité central. Selon le préambule figurant dans le RE, l'AD se considère «comme un organe de la FMH agissant de manière autonome» et «un organe de liaison et de communication entre le CC et la Chambre médicale ainsi qu'un organe «correcteur», dans le sens d'une séparation des pouvoirs entre l'exécutif et la base du corps médical. Sa position entre le CC et la Chambre médicale permet d'assurer une coopération optimale entre les organes du corps médical prenant les décisions finales». On rappellera que conformément aux statuts révisés en mai et en décembre 2006, l'AD prend la succession de l'ancienne Conférence des présidents. Comme cette dernière, elle est chargée de traiter toutes les «questions importantes de politique sanitaire et professionnelle». Mais l'AD peut prendre des décisions, ce que la Conférence des présidents ne pouvait pas faire en sa qualité d'organe consultatif des décisions portant par exemple sur l'adhésion à un comité d'initiative ou sur l'approbation de conventions tarifaires nationales.

- La nouvelle Commission de gestion (CdG) succède à l'ancienne Commission des finances. Elle a pour mission de contrôler «la gestion de l'AD, du CC et du SG (Secrétariat général)», alors que la Commission des finances ne devait, pour l'essentiel, que veiller au respect du budget.

Quelle sera la suite du processus?

En décembre 2007, un groupe de travail dirigé par le Dr Peter Wiedersheim (Saint-Gall) soumettra sa proposition de nouveaux modèles de direction à la Chambre médicale. Selon toute probabilité, les nouvelles règles du jeu applicables au sein de la FMH devraient donc être entièrement fixées en décembre 2007.

\section{Tout est-il gravé à jamais dans la pierre?}

Non. Le président et le Comité central ont toujours indiqué clairement que les nouvelles structures devraient être utilisées et essayées pendant environ deux ans. Le corps médical pourra ensuite décider de ce qui a fait ses preuves et, le cas échéant, de ce qu'il souhaite ajuster ou changer au vu des expériences faites.

Hanspeter Kuhn, avocat, secrétaire général adjoint de la FMH 